\title{
ON A PERIODICALLY FORCED, WEAKLY DAMPED PENDULUM. PART 2: HORIZONTAL FORCING
}

\author{
PETER J. BRYANT ${ }^{\prime}$ AND JOHN W. MILES ${ }^{2}$
}

(Received 12 May 1989; revised 18 October 1989)

\begin{abstract}
We consider the phase-locked solutions of the differential equation governing planar motion of a weakly damped pendulum driven by horizontal, periodic forcing of the pivot with maximum acceleration $\varepsilon g$ and dimensionless frequency $\omega$. Analytical solutions for symmetric oscillations at smaller values of $\varepsilon$ are continued into numerical solutions at larger values of $\varepsilon$. A wide range of stable oscillatory solutions is described, including motion that is symmetric or asymmetric, downward or inverted, and at periods equal to the forcing period $T \equiv 2 \pi / \omega$ or integral multiples thereof. Stable running oscillations with mean angular velocity $p \omega / q$, where $p$ and $q$ are integers, are investigated also. Stability boundaries are calculated for swinging oscillations of period $T, 2 T$ and $4 T ; 3 T$ and $6 T$; and for running oscillations with mean angular velocity $\omega$. The period-doubling cascades typically culminate in nearly periodic motion followed by chaotic motion or some independent periodic motion.
\end{abstract}

\section{Introduction}

We consider a pendulum that is forced by a horizontal acceleration $\varepsilon g \sin \omega t$ of its pivot. The equation of motion is

$$
\ddot{\theta}+2 \delta \dot{\theta}+\sin \theta=\varepsilon \cos \theta \sin \omega t,
$$

where $\theta$ is the angular displacement from the downward vertical, $\delta$ is the damping ratio (actual/critical), $\omega$ is the ratio of the forcing frequency to the natural frequency, and the unit of time is the inverse natural frequency. We assume $\delta<1, \varepsilon=O(1)$ and $\omega=O(1)$ in the analytical formulation based

\footnotetext{
${ }^{1}$ Department of Mathematics, University of Canterbury, Christchurch, New Zealand.

${ }^{2}$ Institute of Geophysics and Planetary Physics, University of California at San Diego, La Jolla, California 92093, U.S.A.

(C) Copyright Australian Mathematical Society 1990, Serial-fee code 0334-2700/90
} 
on a sinusoidal approximation for $\theta$, and set $\delta=1 / 8$ in the numerical calculations. Resonance curves are defined as plots of $\langle E\rangle^{\frac{1}{2}}$ vs $\omega$, where

$$
E=\dot{\theta}^{2} / 2+1-\cos \theta
$$

is a measure of the energy of oscillation. This investigation follows one into the phase-locked solutions of a weakly damped pendulum forced by a periodic tôrque [1], and precedes ânother into fórciñg by vertical periódic acceleration of the pendulum pivot [2].

The $T$-periodic, symmetric solutions of (1.1) may be approximated by

$$
\theta=\alpha \sin (\omega t-\phi),
$$

where the amplitude $\alpha=\alpha(\omega ; \delta, \varepsilon)$ and the phase $\phi=\phi(\omega ; \delta, \varepsilon)$ are to be determined through an appropriate averaging, and one of our aims is to find the domain of validity of this approximation (it turns out to be roughly $\varepsilon \lesssim 0.8$ ). We also aim to illustrate the richness and variety of phaselocked solutions of (1.1) that lie outside of the domain of (1.3), or any other simple analytical approximation, and require numerical analysis for their elucidation. This is based on numerical collocation applied to truncated Fourier expansions for $\theta(t)$ [1]. A systematic numerical search was made of the asymptotic solutions in time of (1.1), using step-by-step integration with a local error tolerance of $10^{-10}$, to complement the Fourier series method. The chaotic solutions of (1.1) were not investigated further, since our primary focus is on the bifurcation structure.

We begin, in Section 2, by calculating the resonance curves for $T$-periodic, symmetric solutions of $(1.1)$ through both the analytical approximation (1.3) and numerical analysis. If $\varepsilon>\varepsilon_{c}(\delta)\left[\varepsilon_{c}(1 / 8)=0.516\right]$ the resonance curve is triple-valued between two turning points, $\omega_{ \pm}\left(\omega_{+}>\omega_{-}\right)$, at which the gradient is vertical, and the part that connects the turning points comprises only unstable states. If $\varepsilon<\varepsilon_{x}=4.63 \delta$ the resonance curve is of the conventional type, tilted to the left with a single maximum (see Figure 1a), but if $\varepsilon>\varepsilon_{x}$ the curve crosses itself near $\omega=0.80$ and executes a closed loop (see Figures 1b,c). This crossing does not appear to have been previously reported, and is a point where two distinct solutions have the same values of $\varepsilon, \omega$ and $\langle E\rangle^{\frac{1}{2}}$.

The symmetric solutions lose stability through either a turning-point (saddle-node) or a symmetry-breaking (pitchfork) bifurcation, but were not found to lose stability through period-doubling or Hopf bifurcations [10]. The turning-point bifurcations, which appear at $\omega_{ \pm}$(see above) and are associated with the well known tuning hysteresis of a nonlinear oscillator, typically imply a transition between symmetric states, and are implicit in the symmetric solution. The symmetry-breaking bifurcations appear in pairs for 


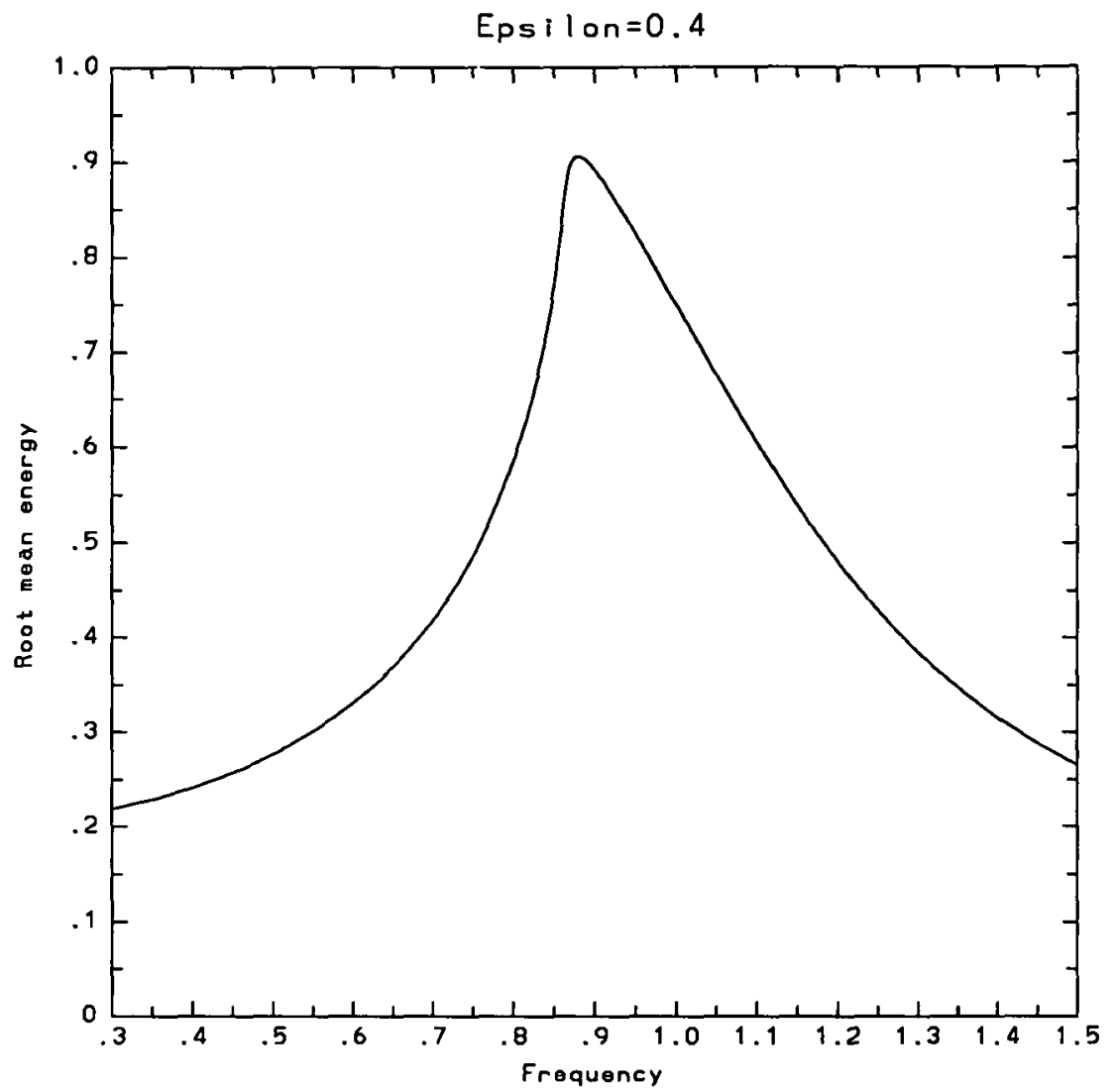

Figure la. The resonance curve for symmetric oscillations with $\delta=1 / 8$ and $\varepsilon=0.4$. The analytically and numerically calculated curves coincide.

$\varepsilon>\varepsilon_{s}(\delta)\left[\varepsilon_{s}(1 / 8)=1.022\right]$, and we determine the symmetry-breaking locus in the $\varepsilon, \omega$ plane. The $T$-periodic, asymmetric solutions are obtained through an analytical approximation in which a constant $\theta_{0}$ is added to the right of (1.3), and through numerical analysis with both odd and even harmonics admitted in the Fourier series. The resulting analytical approximation is only qualitatively valid, and all subsequent calculations are based on numerical analysis.

The $T$-periodic, asymmetric solution loses stability to a $2 T$-periodic, asymmetric solution through a period-doubling bifurcation, which is followed by a $4 T$-periodic, asymmetric solution through a second period-doubling bifurcation, and so on through a period-doubling cascade to chaos (or to independent, stable, periodic oscillations). We calculate the period-doubling, quadrupling, and octupling loci in the $\varepsilon, \omega$ plane, and the resonance curves 
Epsiton $=0.8$

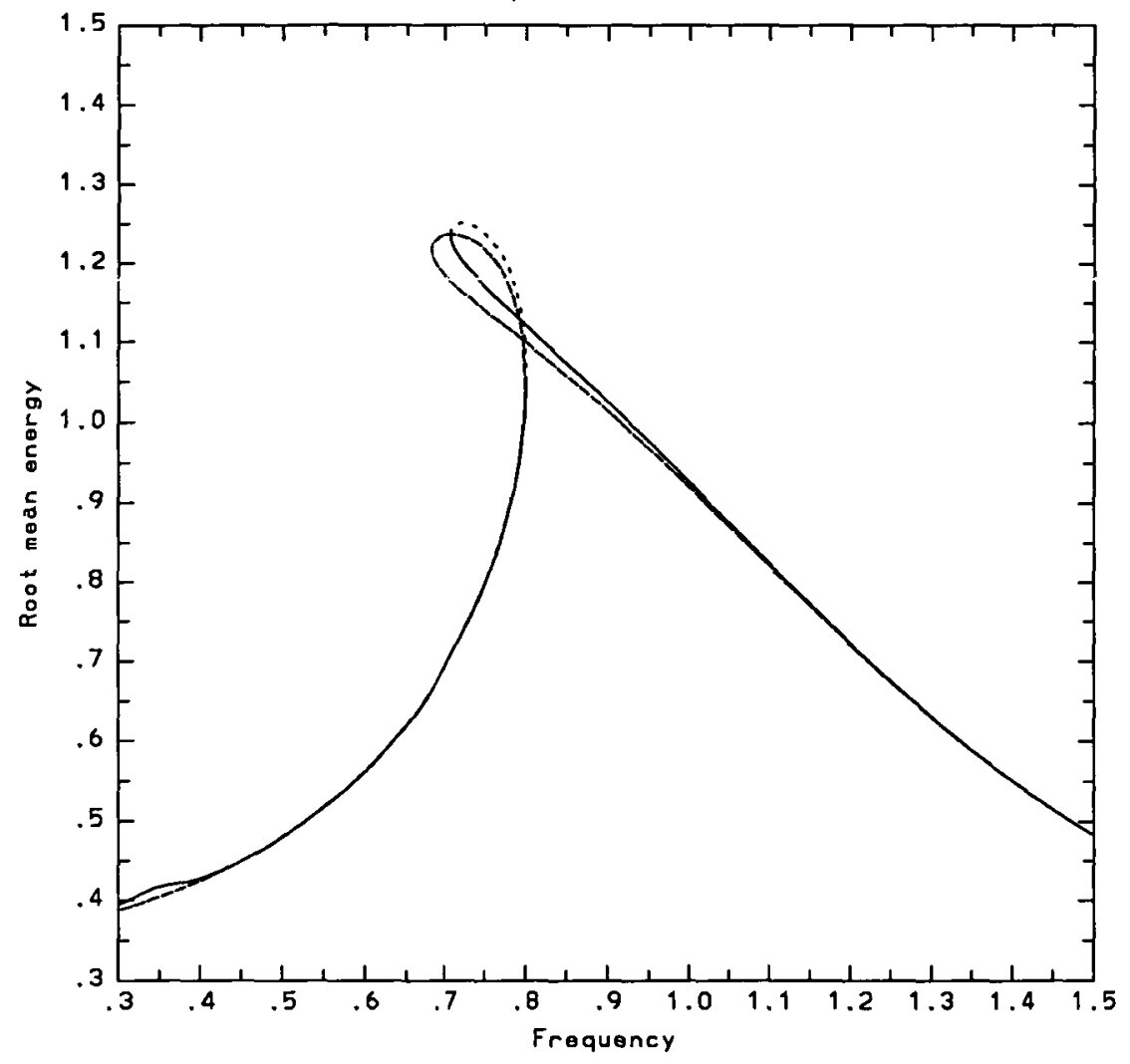

FIGURE $1 \mathrm{~b}$. The resonance curves for symmetric oscillations with $\delta=1 / 8$ and $\varepsilon=0.8$. The oscillations are stable on the solid sections and unstable on the dotted sections. The numerically calculated curve is solid or dotted, and the approximate analytical curve is dashed.

for $2^{n} T$-periodic oscillations for $n=0,1,2,3$ and 4 .

We find, as did D'Humieres et al [4] for the torque driven pendulum, and as is to be expected for any nonlinear oscillator with a symmetric potential (cf. [9]), that (1.1) also admits stable symmetric solutions of period $3 T$ ( $\mathrm{sim}$ ilar oscillations of period $5 T, 7 T, \ldots$ also occur in smaller intervals of $\varepsilon$ and $\omega)$. As $\varepsilon$ is increased these oscillations lose stability to $3 T$-periodic, asymmetric oscillations through a symmetry-breaking bifurcation, which, in turn, lose stability to $6 T$-periodic oscillations through a period-doubling bifurcation, and so on through a $12 T, 24 T, \ldots$ period-doubling cascade to chaos (or to independent, stable, periodic oscillations). We report the details in Section 3. 
Epsilon $=1.2$

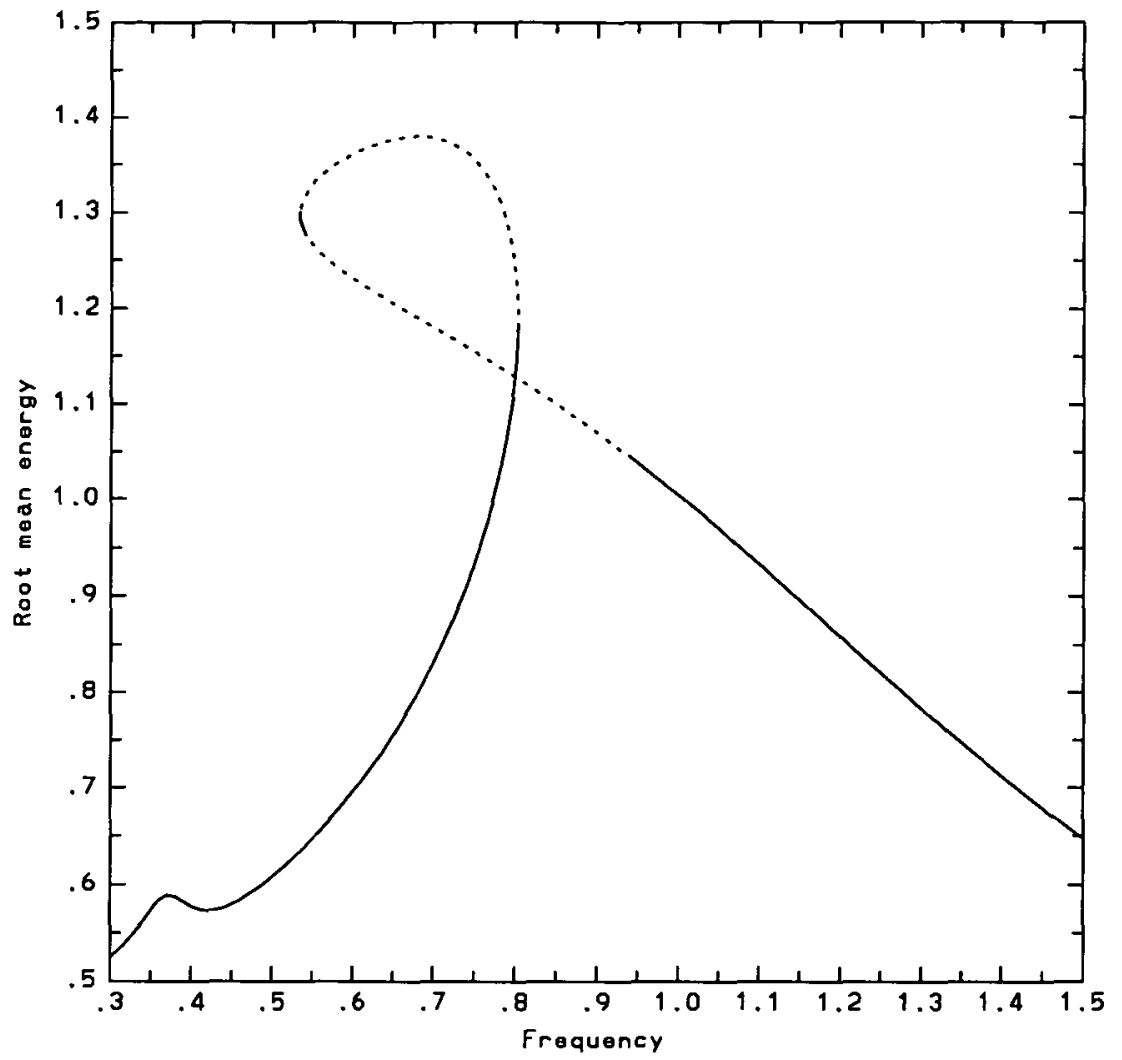

FIGURE 1c. The numerically calculated rsonance curve for symmetric oscillations, with $\delta=$ $1 / 8$ and $\varepsilon=1.2$. The oscillations are stable on the solid sections and unstable on the dotted sections.

The torque-driven pendulum executes stable, $T$-periodic, inverted $(\langle\theta\rangle=$ $\pi$ ) oscillations in a finite, $\varepsilon, \omega$ domain $[1,8]$, but the only $T$-periodic, inverted oscillations that we find in the present case are unstable. We do, however, find stable, $3 T$-periodic, inverted oscillations, which we report in Section 3. These solutions follow a symmetry-breaking, period-doubling sequence similar to that described above for the normal (downward) oscillations, although the two families appear to be independent.

A large number of running oscillations with $\langle\dot{\theta}\rangle=p \omega / q$ is possible, just as for the torque-driven pendulum. Their stability domains typically decrease with increasing $p$ and $q$, but we did obtain one such solution for $p=3$ and $q=46$. The family with the largest stability domain is that for $p=1$ and $q=1 \quad(\langle\dot{\theta}\rangle=\omega)$, which we examine in Section 4 . 
We determined the stability of our oscillatory solutions through the numerical integration of (1.1) with initial conditions in the neighbourhood of the solution to be tested. We also attempted an analytical stability analysis for the analytical approximations in Section 2, following the perturbation procedure (leading to Hill's equation) that proved moderately successful for the torque driven pendulum [7], but the results proved to be reliable only for the turning-point changes of stability of the $T$-neriodic, symmetric solutions.

The stability boundaries have a remarkable similarity in shape. In each family of symmetry-breaking and period-doubling solutions, the boundaries tend towards a common curve as $\omega$ decreases. As $\omega$ increases from the common curve, each member of the family passes through a minimum and then increases, usually with positive curvature throughout. This similarity suggests that there may be simple relations between the different stability boundaries for each family of solutions, and also between corresponding stability boundaries for different families of solutions. No such simple relations have been found yet.

The present problem is a much simplified model for the sloshing of a fluid in a container that is subjected to periodic horizontal forcing. Miles [5, 6] used an averaged Lagrangian method to investigate the weakly nonlinear resonant response of the free surface of a fluid in circular and in rectangular horizontally oscillating containers. Planar and nonplanar oscillations of the free surface can occur, and even with the planar case there is an infinite sequence of possible resonating modes of fluid oscillation. One reason for the present investigation into the planar oscillations of a forced pendulum is that there is then only one resonant mode of oscillation, but as is indicated above, a large variety of types of nonlinear oscillation is still possible even for this simple model.

\section{T-periodic oscillations and their descendants}

We represent $T$-periodic solutions of (1.1) by the Fourier series

$$
\theta=\sum_{k=0}^{N}\left(a_{k} \cos k \omega t+b_{k} \sin k \omega t\right),
$$

where either $a_{0}, \ldots, b_{N}$ and $N$ are determined numerically as described above or the series is truncated at $N=1$ and recast in the form

$$
\theta=\theta_{0}+\alpha \sin \tau\left(-\pi<\theta_{0} \leq \pi, \alpha>0\right), \quad \tau=\omega t-\phi,
$$

to determine analytical approximations. Substituting (2.2a,b) into (1.1) and 
taking moments with respect to $1, \cos \tau$, and $\sin \tau$, we obtain

$$
\begin{gathered}
\left(J_{0}+\varepsilon J_{1} \cos \phi\right) s_{0}=0, \\
2 \delta \alpha \omega=\varepsilon\left(J_{0}+J_{2}\right) c_{0} \sin \phi, \\
\alpha\left[\left(J_{0}+J_{2}\right) c_{0}-\omega^{2}\right]=\varepsilon\left(J_{0}-J_{2}\right) c_{0} \cos \phi,
\end{gathered}
$$

where, here and subsequently, $J_{n}=J_{n}(\alpha)$ is a Bessel function, $c_{0}=\cos \theta_{0}$, and $s_{0}=\sin \theta_{0}$. We note that (2.3) may be derived through an averageLagrangian formulation (cf. [7]).

\subsection{Symmetric oscillations}

We obtain symmetric oscillations by setting $a_{2 j}=b_{2 j}=0,(j=0,1$, $2, \ldots)$ in $(2.1)$ and $\theta_{0}=0$ in $(2.2)$ and (2.3). Eliminating $\phi$ between $(2.3 \mathrm{~b}, \mathrm{c})$ and solving for $\omega^{2}$, we obtain the resonance curve(s)

$$
\omega^{2}=\Omega-2 \delta^{2} X^{2} \pm X\left[(\varepsilon \Omega / \alpha)^{2}-4 \delta^{2} \Omega+4 \delta^{4} X^{2}\right]^{\frac{1}{2}}\left(\theta_{0}=0\right),
$$

where

$$
\Omega(\alpha) \equiv J_{0}+J_{2}=\frac{2 J_{1}}{\alpha}=1-\frac{1}{8} \alpha^{2}+\frac{\alpha^{4}}{192}+\cdots,
$$

and

$$
X(\alpha) \equiv \frac{J_{0}-J_{2}}{J_{0}+J_{2}}=\frac{\alpha J_{1}^{\prime}}{J_{1}}=1-\frac{1}{4} \alpha^{2}-\frac{\alpha^{4}}{96}+\cdots .
$$

The corresponding approximation to the mean energy, obtained through the substitution of $\theta=\alpha \sin \tau$ into (1.2), is

$$
\langle E\rangle=\alpha^{2} \omega^{2} / 4+1-J_{0},
$$

and the resonance curves, $\langle E\rangle^{\frac{1}{2}}$ vs $\omega$, are determined by (2.4) and (2.7).

The two branches (2.4) join at the smallest zero of the radical, $\alpha=\alpha_{m}$ which is a maximum of $\alpha$ vs $\omega$ and is determined by

$$
2(\delta \alpha / \Omega)\left(\Omega-\delta^{2} X^{2}\right)^{\frac{1}{2}}=\varepsilon \quad\left(\alpha=\alpha_{m}\right) .
$$

If $\alpha_{m}<\alpha_{1}^{\prime}=1.841$, the smallest zero of $J_{1}^{\prime}$ (and therefore of $X$ ), for which a necessary and sufficient condition is $\varepsilon>4.63 \delta \equiv \varepsilon_{x}$, the two branches cross at $\alpha=\alpha_{1}^{\prime}$ and $\omega^{2}=\Omega\left(\alpha_{1}^{\prime}\right)$ or, equivalently, $\omega=0.795$ and $\langle E\rangle^{\frac{1}{2}}=1.104$. The crossing, which is common to all resonance curves for which $\varepsilon>\varepsilon_{x}$, differs significantly from that associated with the breaking of the resonance curve for $\varepsilon>\varepsilon_{*}$ (cf. [7]), where $\varepsilon_{*}$ is the largest value of $\varepsilon$ for which the radical in (2.4) admits a real zero for $\alpha=\alpha_{m}$ and lies outside of the domain of the present approximation.

The resonance curve for symmetric oscillations with $\delta=1 / 8$ and $\varepsilon=0.4$ is plotted in Figure 1a. The analytical approximation implied by (2.4) and 
(2.7) coincides with the numerical result. The curve is single-valued in $\langle E\rangle^{\frac{1}{2}}$, and the corresponding symmetric oscillations are everywhere stable.

The approximate analytical (dashed) and numerical (solid or dotted) resonance curves for symmetric oscillations with $\delta=1 / 8$ and $\varepsilon=0.8$ are plotted in Figure 1b. The two curves differ at the larger values of $\langle E\rangle^{\frac{1}{2}}$, where the neglect of the higher harmonics in $(2.2 \mathrm{~b})$ becomes significant. The analytical approximation aiso misses the super-harmonic resonance at $\omega=i / 3$, which arises from the resonant forcing of the third harmonic. The resonance curve is triple-valued in $\langle E\rangle^{\frac{1}{2}}$ between the two turning points at $\omega=\omega_{-}=0.707$ and $\omega=\omega_{+}=0.800$, and the symmetric oscillations are unstable on the dotted segment of the curve connecting the turning points. The symmetric oscillations are stable on all solid segments of the curve.

The resonance curve for symmetric oscillations with $\delta=1 / 8$ and $\varepsilon=1.2$ is plotted in Figure 1c. As in Figure 1b, symmetric oscillations are stable on the solid segment to the lower turning point at $\omega=\omega_{+}=0.803$ and then unstable on the dotted segment to the upper turning point at $\omega=\omega_{-}=0.533$. However, in contrast to Figure 1b, the symmetric oscillations in Figure 1c are stable only for a small solid section below the upper $\left(\omega=\omega_{-}\right)$turning point, are unstable for a much larger dotted section, and are again stable as $\omega$ increases further. Asymptotic solutions of (1.1) on the unstable sides of the latter two stability boundaries show that both are symmetry-breaking bifurcations, with the symmetric oscillations losing stability to asymmetric oscillations. As $\varepsilon$ is increased from 1.2 to 2.0 the resonance curves for symmetric oscillations retain the same form as in Figure 1c, with the short stable section just below the upper turning point becoming vanishingly small, and the unstable section following it increasing in length at both ends.

We determine analytical approximations to the turning points, $\omega=\omega_{ \pm}$ and $\alpha=\alpha_{ \pm}$, where $\omega_{+}>\omega_{-}$and $\alpha_{+}<\alpha_{-}$, by calculating $d \omega / d \alpha=0$ from (2.4). Invoking $\delta^{2} \ll 1$, we obtain

$$
\frac{2 \alpha J_{2}}{J_{0}-3 J_{2}+\alpha^{2} \Omega}=\varepsilon, \quad \omega_{+}^{2}=\Omega-\frac{\varepsilon X \Omega}{\alpha}\left(\alpha=\alpha_{+}\right),
$$

wherein $O\left(\delta^{2}\right)$ error terms are implicit, for the determination of the lower turning point. The upper turning point is determined by

$$
\alpha_{-}=\alpha_{m}\left[1-1 / 2 \delta^{2} X^{2}\left(\frac{\Omega+J_{2}}{J_{2}^{2}}\right)\right]_{m}, \quad \omega_{-}^{2}=\Omega_{m}-\delta^{2} X_{m}^{2}\left(\frac{J_{0}}{J_{2}}+4\right)_{m} \text {, }
$$

wherein $O\left(\delta^{4}\right)$ error terms are implicit, and the subscript $m$ implies evaluation at $\alpha=\alpha_{m}$, which is determined by (2.8). The loci of the turning points are plotted in Figure 2, with the approximate analytical values from (2.9) and (2.10) shown as dashed curves. The solid curve on the right of Figure 


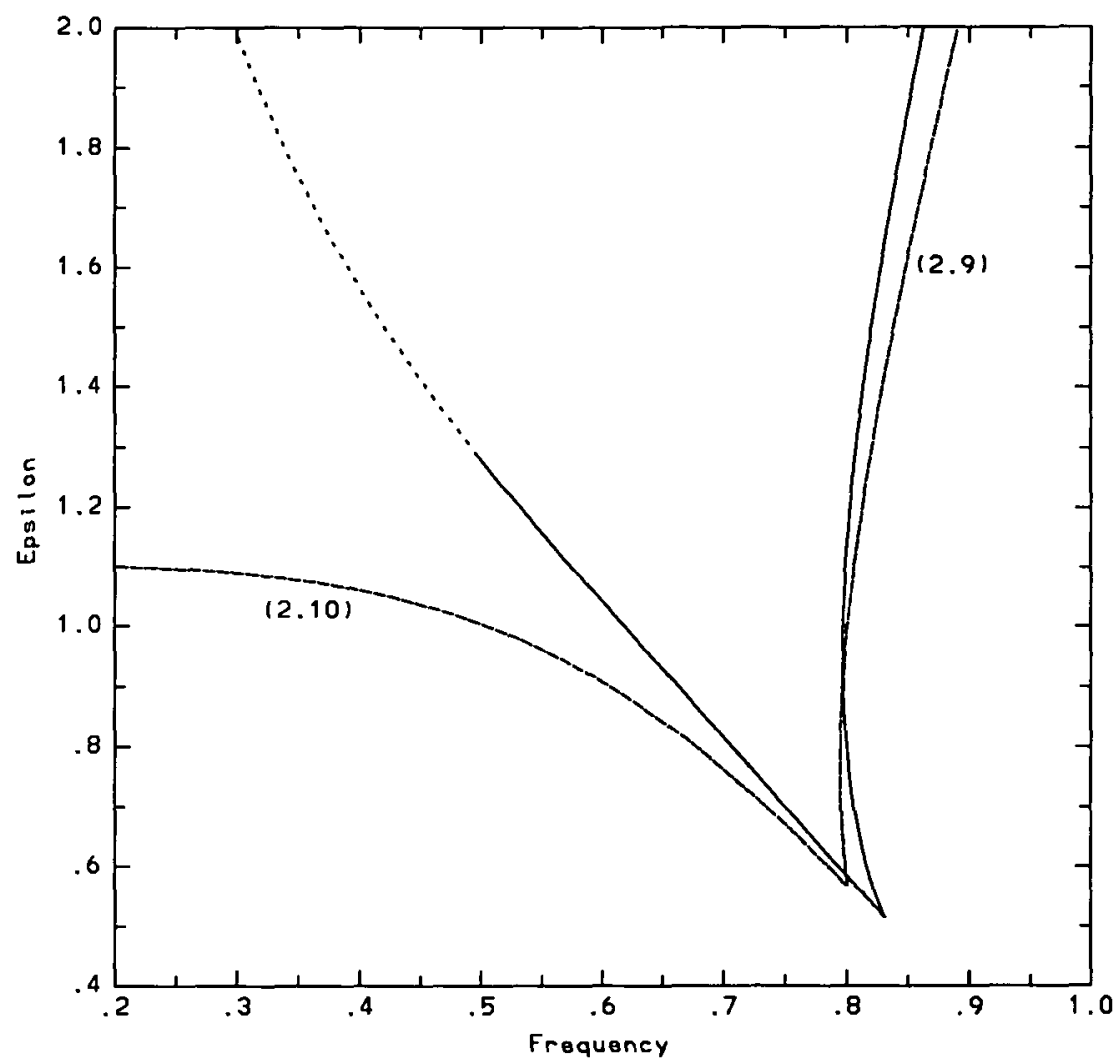

Figure 2. The turning-point curves for symmetric oscillations with $\delta=1 / 8$. The solid and dotted curves are the numerically calculated results; the dashed curves are the approximate analytical results $(2.9),(2.10)$.

2 is the numerically determined locus for $\omega_{+}$with which (2.9) shows good agreement (presumably by virtue of the modest contributions of the higher harmonics at the lower turning point). The curve on the left of Figure 2, which is solid on the lower part and dotted on the upper part, is the numerically determined locus for $\omega_{-}$. The lower, solid part is a stability boundary for symmetric oscillations in $\omega>\omega_{-}$in at least a small neighbourhood of $\omega_{-}$. In the upper, dotted part the stable region for $\omega>\omega_{-}$has a width less than $10^{-3}$ in $\omega$ and is difficult to detect. The approximate analytical result $(2.10)$ for $\omega_{-}$is in good agreement with the exact numerical curve in the lower part of Figure 2, but it deteriorates above $\varepsilon \simeq 0.8$ in consequence of the increasing importance of higher harmonics on the higher parts of the resonance curves. The turning points coincide for $\varepsilon=\varepsilon_{c}(\delta)$ and are absent for $\varepsilon<\varepsilon_{c}\left[\varepsilon_{c}(1 / 8)=0.516\right]$. 


\subsection{Asymmetric oscillations}

If $s_{0} \neq 0$, so that (2.3a) implies $J_{0}+\varepsilon J_{1} \cos \phi=0, \phi$ may be eliminated among $(2.3 \mathrm{a}, \mathrm{b}, \mathrm{d})$ to obtain

$$
\left(\frac{J_{0}}{J_{1}}\right)^{2}+\left[\frac{2 \delta\left(\alpha J_{1}+X J_{0}\right)}{J_{1} \omega}\right]^{2}=\varepsilon^{2}, \quad c_{0}=\frac{\alpha^{2} \omega^{2}}{2\left(\alpha J_{1}+X J_{0}\right)}
$$

for the determination of $\alpha$ and $c_{0}$ for prescribed $\omega$ and $\varepsilon$. The symmetrybreaking bifurcations $\left(c_{0}=1, \alpha=\alpha_{s}, \omega=\omega_{s}\right)$ are determined by

$$
\frac{J_{0}^{2}+2 \delta^{2} \alpha^{2}\left(\alpha J_{1}+X J_{0}\right)}{J_{1}^{2}}=\varepsilon^{2}, \quad \omega_{s}^{2}=\frac{2\left(\alpha J_{1}+X J_{0}\right)}{\alpha^{2}} \quad\left(\alpha=\alpha_{s}\right) \text {. }
$$

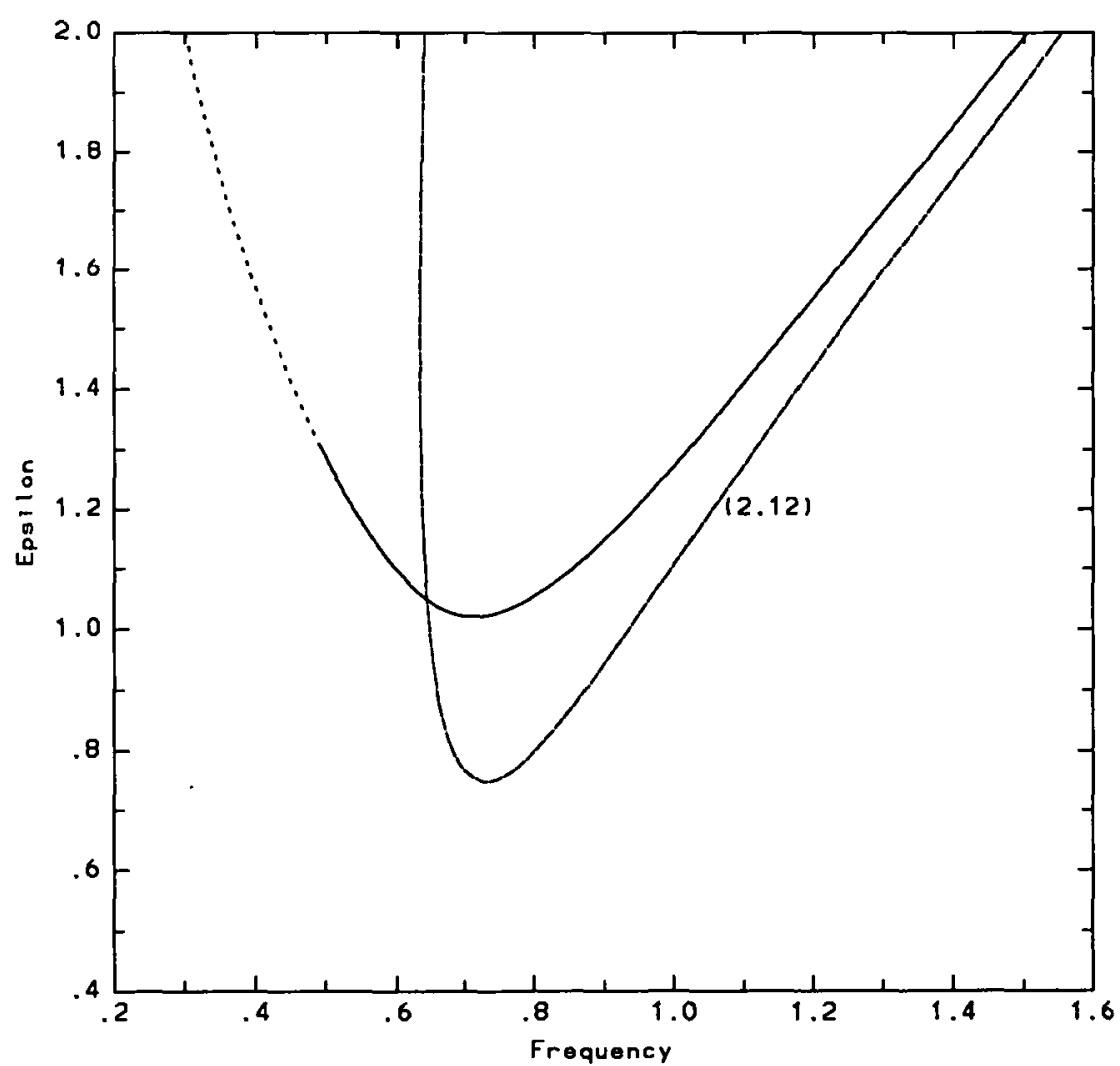

Figure 3. The symmetry-breaking curves for $\delta=1 / 8$. The solid and dotted curve is the exact numerical result, and the dashed curve is the approximate analytical result (2.12). 
The symmetry-breaking curve is plotted in Figure 3, with the analytical approximation $(2.12 \mathrm{a}, \mathrm{b})$ drawn as a dashed curve. The solid symmetry-breaking curve is a stability boundary for symmetric oscillations when $\varepsilon>\varepsilon_{s}(\delta)$ $\left[\varepsilon_{s}(1 / 8)=1.022\right]$, with stable symmetric oscillations on the outside of the curve and stable asymmetric oscillations on the inside of the curve; symmetry breaking is absent if $\varepsilon<\varepsilon_{s}$. The symmetry-breaking curve is dotted where the bandwidth of asymmetry stability becomes so small as to be diffcult to detect. The agreement between the analytical approximation and the numerical curve is reasonable at the larger values of $\omega$, but, as in Figure 2, the analytical approximation is unsatisfactory for the smaller values of $\omega$, where higher harmonics become significant. The failure is more pronounced than in Figure 2 owing to the presence of the second harmonic (which vanishes for the symmetric oscillations). Resonance curves for the asymmetric oscillations begin and end at symmetry-breaking bifurcation points on the resonance curves for symmetric oscillations.

\subsection{Period-doubling}

We represent $m T$-periodic oscillations by the Fourier series

$$
\theta=\sum_{k=0}^{N}\left[a_{k} \cos (k \omega t / m)+b_{k} \sin (k \omega t / m)\right] .
$$

The first period-doubling locus is a stability boundary for $T$-periodic, asymmetric oscillations with stable, $T$-periodic, asymmetric oscillations on the outside of the curve and stable, $2 T$-periodic, asymmetric oscillations on the inside of the curve. Resonance curves for the $2 T$-periodic, asymmetric oscillations begin and end at the period-doubling bifurcation points on the resonance curves for the $T$-periodic, asymmetric oscillations. The second period-doubling locus is a stability boundary for $2 T$-periodic oscillations, with $4 T$-periodic oscillations being stable on the inside of the curve. This sequence continues through a period-doubling cascade, with stable intervals of decreasing bandwidth, followed by nearly periodic motion, then chaotic motion or an independent stable oscillation.

The principal stability boundaries for swinging oscillations of periods $T$, $2 T$ and $4 T$ are summarised in Figure 4. The curves $C_{-}$and $C_{+}$are the turning-point boundaries of Figure 2, while $C_{1}$ is the symmetry-breaking locus of Figure 3. Stable symmetric oscillations are found everywhere to the left of $C_{+}$, and to the right of $C_{-}$below $C_{1}$. There are two independent families of stable symmetric oscillations in the lower $V$-shaped region between $C_{-}$and $C_{+}$, and a common family below the intersection of $C_{-}$ and $C_{+}$. Stable, $T$-periodic, asymmetric oscillations are found between $C_{1}$ and the first period-doubling locus $C_{2}$. Those with period $2 T$ occur be- 


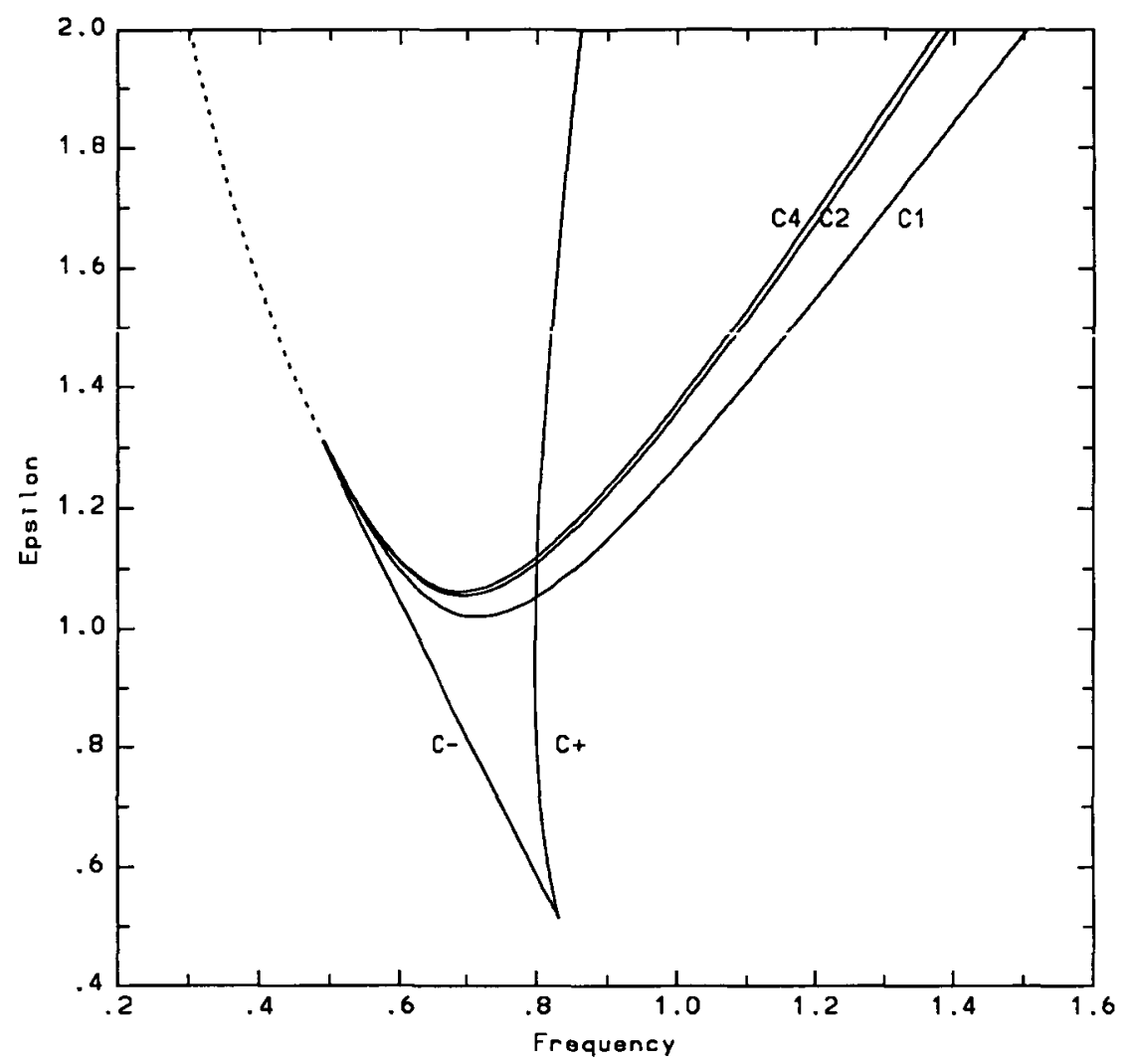

FIGURE 4. Stability boundaries for oscillations of periods $T, 2 T$ and $4 T$, with $\delta=1 / 8$. The stability boundaries $C_{8}, \ldots, C_{\infty}$ are indistinguishable from $C_{4}$ on the scale of the figure. Note the coalescence of the several stability boundaries into a single curve on the left.

tween $C_{2}$ and the second period-doubling locus $C_{4}$. The period-doubling loci $C_{8}, \ldots, C_{\infty}$ are indistinguishable from $C_{4}$ on the scale of the figure.

The symmetry-breaking, period-doubling sequence is demonstrated more explicitly in Figure 5 for $\delta=1 / 8$ and $\varepsilon=1.2$, where resonance curves are drawn for the different forms of swinging oscillation from symmetric with period $T$ to asymmetric with period $8 T$. The oscillations are stable on the solid curves and unstable on the dotted curves. The upper curve is a portion of the resonance curve for symmetric oscillations sketched in Figure 1c. The lower curve, beginning at the symmetry-breaking point, is a portion of the resonance curve for $T$-periodic asymmetric oscillations. The resonance curve labelled $2 T$ for $2 T$-periodic oscillations begins at the first period-doubling point. It bifurcates to the resonance curve labelled $4 T$ for $4 T$-periodic oscillations, then to the unlabelled resonance curve for $8 T$-periodic oscillations. 
Epsilon $=1.2$

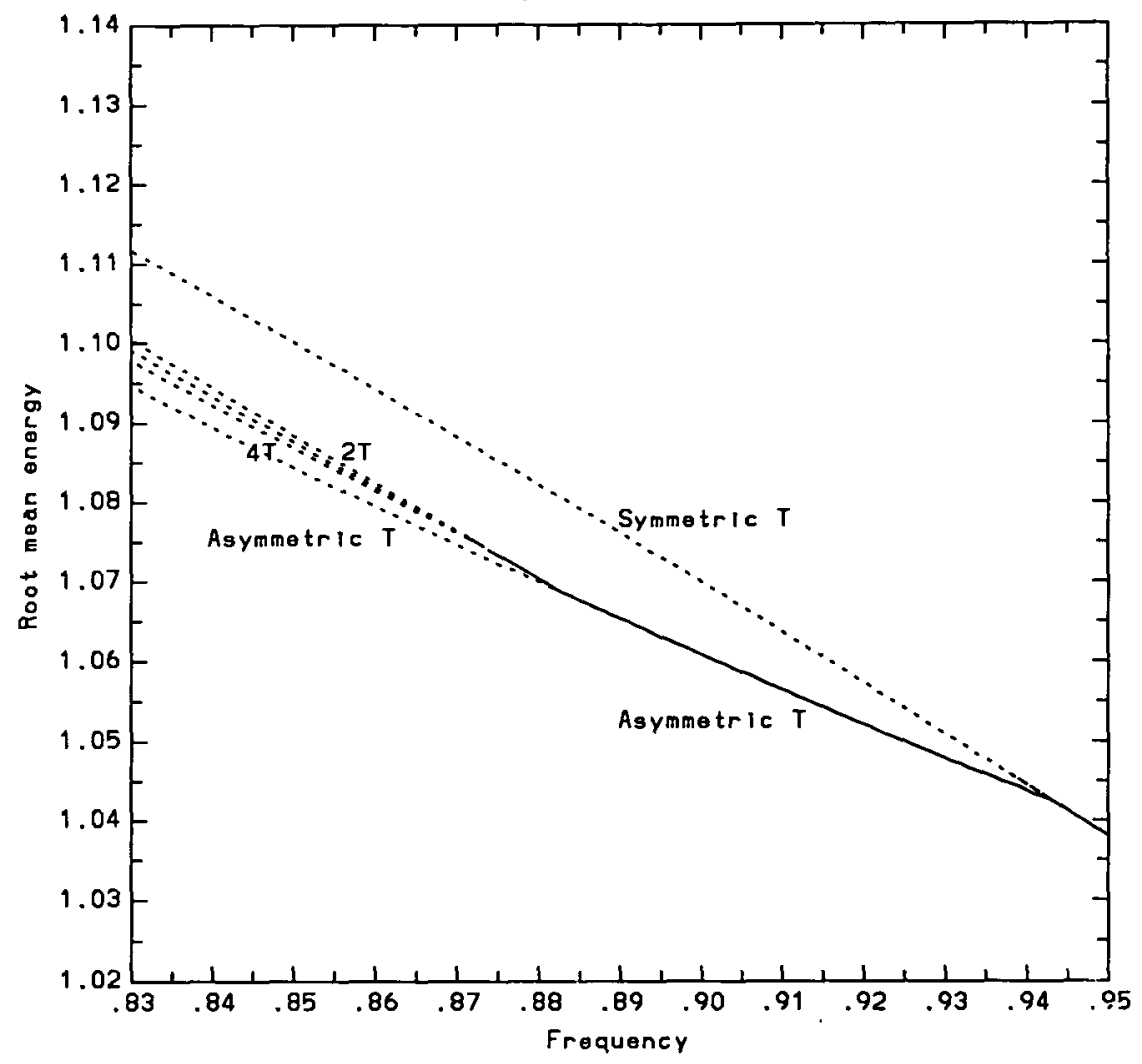

FIGURE 5. Resonance curves for the symmetry-breaking, period-doubling sequence of periods $1,2,4$ and 8 times the forcing period, with $\delta=1 / 8$ and $\varepsilon=1.2$.

The resonance curves appear to be leapfrogging towards a limiting curve as the period multiplicity increases.

The bifurcation from $2 T$-periodic oscillations to $4 T$-periodic oscillations in Figure 5 occurs at $\omega=0.8733$, that from $4 T$-periodic oscillations to $8 T$ periodic oscillations at $\omega=0.8712$, and that from $8 T$-periodic oscillations to $16 T$-periodic oscillations at $\omega=0.8708$. The ratio of these two frequency bandwidths is 5.25 , in reasonable agreement with Feigenbaum's universal ratio $\delta=4.67$ [3]. Feigenbaum's ratio is derived for one-parameter systems, but ratios here can be calculated for either $\omega$-bandwidths or $\varepsilon$ bandwidths in Figure 4 . If the bandwidth ratio is applicable to the remainder of the period-doubling sequence, the sequence should terminate before $\omega=0.8707$. When $\omega$ is reduced to 0.8707 and further to 0.870 or 0.869 , the oscillations remain nearly periodic in the neighbourhood of the exactly periodic motion. This type of behaviour was observed by D'Humieres et al 
[4] for the torque-driven pendulum, but they interpreted it as an intermittent movement between two unstable states. The present numerical evidence indicates that period-doubling cascades do not culminate in a sharp transition to chaos, but in nearly periodic motion followed by chaos (or by a transition to some independent stable oscillation). The nature of this nearly periodic motion over 500 forcing periods is unchanged when the local error tolerance in the step-by-step integrations of $(1.1)$ is reduced from $10^{-10}$ to $10^{-11}$, but even higher precision may be needed to investigate it fully.
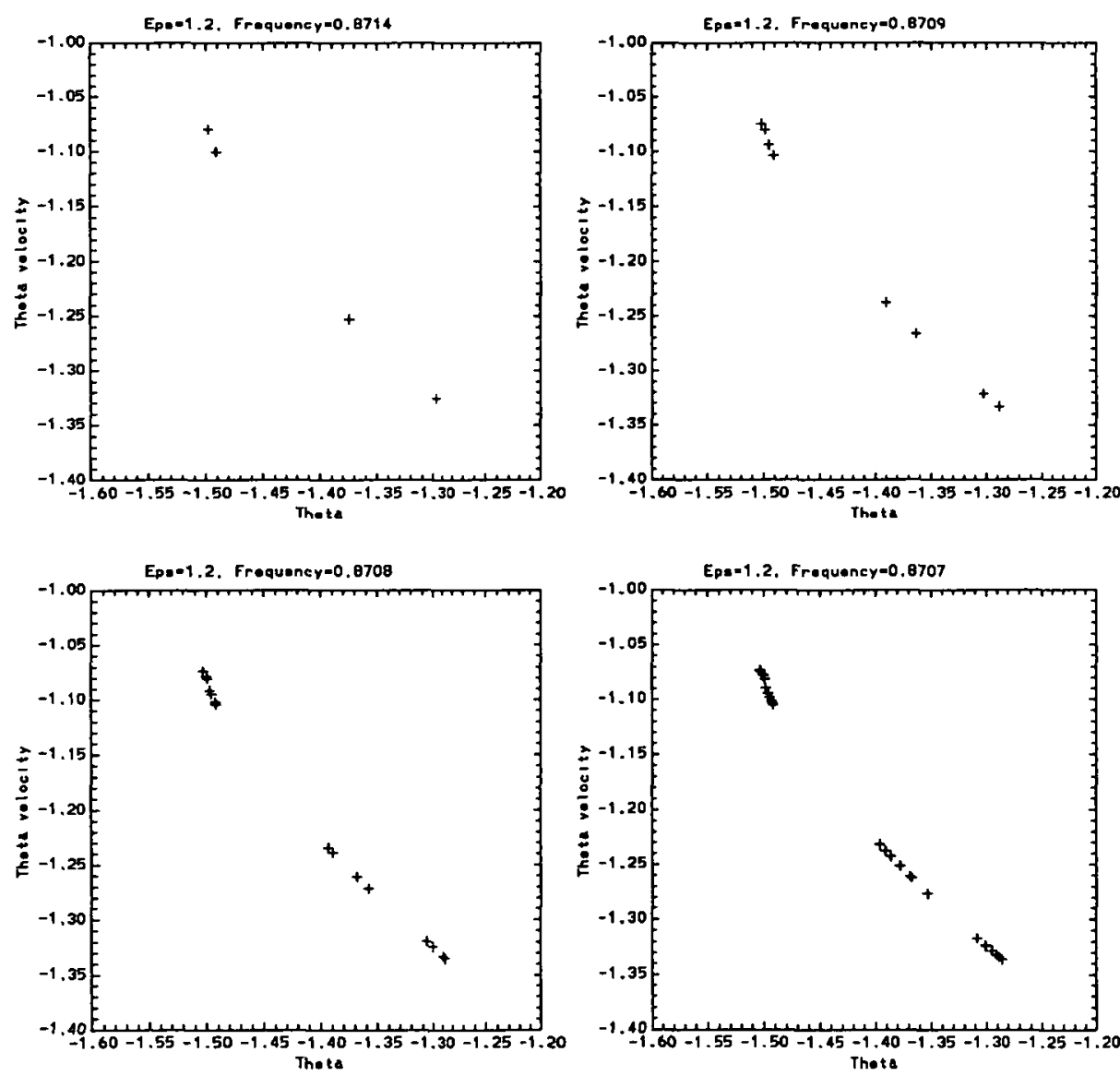

Figure 6. Poincare cross-sections in the $(\theta, \dot{\theta})$ plane for the period-doubling sequence $4 T$, $8 T, 16 T$ and the nearly periodic oscillation after the end of the sequence, with $\delta=1 / 8$ and $\varepsilon=1.2$. 
The sequence described above is illustrated in Figure 6 by Poincare crosssections in the $(\theta, \dot{\theta})$ plane. The first cross-section, at $\omega=0.8714$, shows the locations in this plane of the $4 T$-periodic stable oscillation at times $0, T, 2 T, 3 T, \ldots$. The second cross-section, at $\omega=0.8709$, shows how each of the locations of the previous $4 T$-periodic oscillation bifurcates into two in an $8 T$-periodic stable oscillation. The same bifurcation occurs again to the $16 T$-periodic stable oscillation at $\omega=0.8708$, in the third crosssection. The fourth cross-section, at $\omega=0.8707$, illustrates the nearly periodic motion over 100 forcing periods. The points lie in the neighbourhood of those in the previous cross-sections, and the 100 points are not all distinct (to the resolution of the figure), indicating a motion which is close to the previous exactly periodic oscillations.

\section{3. $3 T$-periodic oscillations and their descendants}

We turn now to $3 T$-periodic, symmetric oscillations of the form

$$
\theta=\sum_{j=1}^{N}\left(a_{2 j-1} \cos [(2 j-1) \omega t / 3]+b_{2 j-1} \sin [(2 j-1) \omega t / 3]\right)
$$

Resonance curves for these oscillations have multiple turning and crossing points, especially for larger $\varepsilon$. The symmetric oscillations bifurcate to stable, $3 T$-periodic, asymmetric oscillations of the form (2.13) with $m=3$. Their resonance curves begin and end at the symmetry-breaking bifurcation points on the resonance curves for the symmetric oscillations, and may have both turning points and crossing points.

The next stability boundary is a period-doubling boundary, across which stable, $6 T$-periodic asymmetric oscillations may be found. The perioddoubling cascade then continues to $12 T$, and so on. The cascade culminates in nearly periodic motion, with the local periodicity lying close to multiples of $3 T$, followed by chaos (or some independent periodic oscillation), much as in the scenario described in the last two paragraphs of Section 2.

The stability diagram for $3 T$-periodic downward oscillations is drawn in Figure 7. The stability boundaries are of the same form as the corresponding boundaries in Figure 4, with $C_{0}$ denoting the turning-point boundary, $C_{3}$ the symmetry-breaking boundary, and $C_{6}$ the first period-doubling boundary. The stable, $3 T$-periodic, symmetric and asymmetric oscillations lie, respectively, between $C_{0}$ and $C_{3}$ and between $C_{3}$ and $C_{6}$. The next perioddoubling boundary, $C_{12}$, is indistinguishable from $C_{6}$ on the scale of Figure 


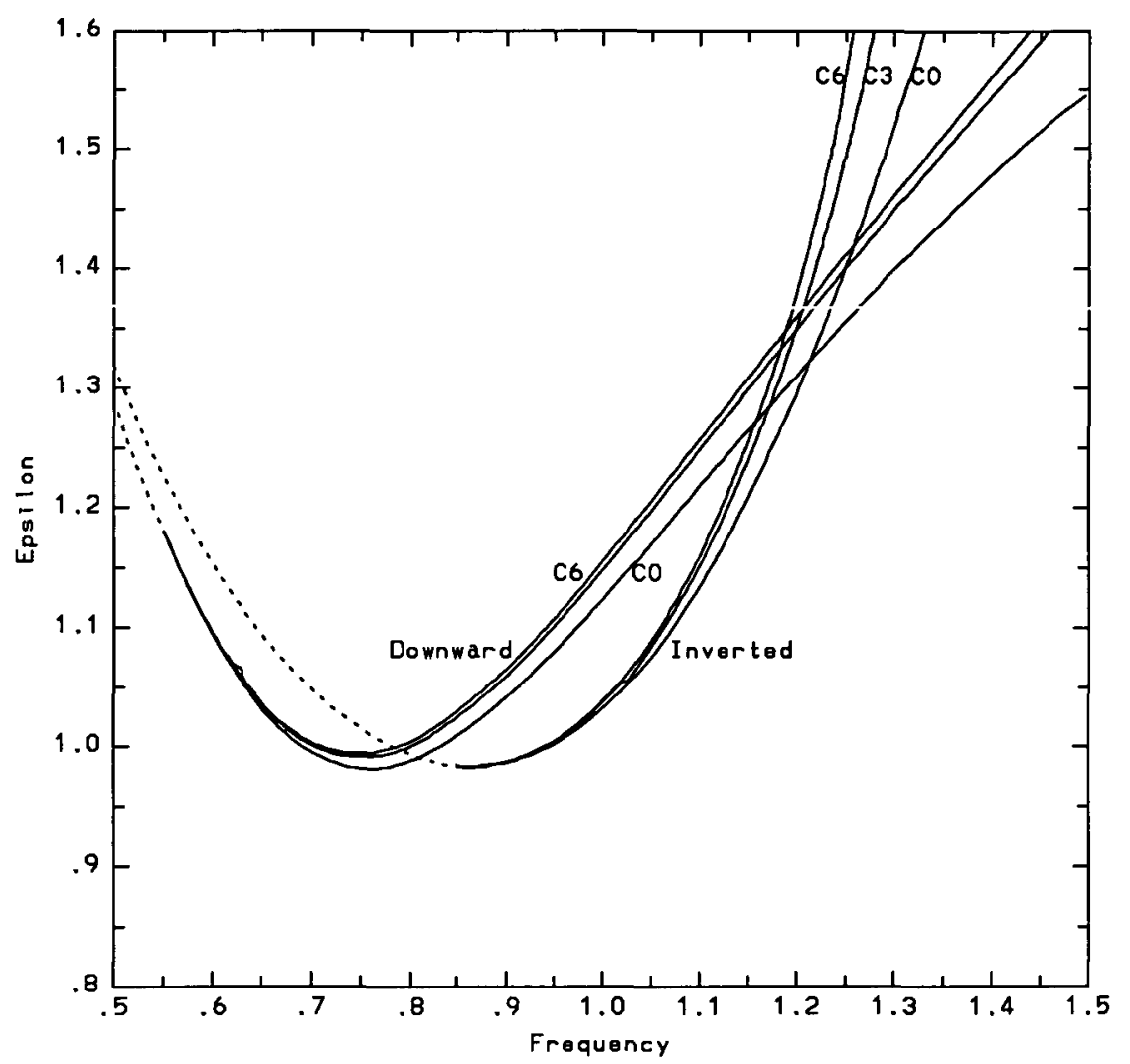

FIGURE 7. Stability boundaries for swinging and inverted oscillations of periods $3 T$, $6 T, \ldots$, with $\delta=1 / 8$.

7. We remark that the regions of stability in Figure 7 overlap significantly those in Figure 4. This implies that the asymptotic state-in particular both period and symmetry - of the motion depends on the initial conditions. The present numerical evidence implies that a sharp transition to chaos occurs when the turning-point stability boundary $C_{0}$ in Figure 7 is crossed from the stable side to the unstable side (in the absence of some independent stable oscillation there). This sharp transition to chaos does not occur in Figure 4 because the unstable side of the turning-point stability boundary for one family of symmetric oscillations is a stable region for the other family of symmetric oscillations.

We reported above that we could find no stable, inverted, $T$-periodic oscillations for the horizontally driven pendulum; however, we did find stable inverted oscillations at higher odd multiples of $T$ and describe those of $3 T$ 
here. The symmetric oscillations have the form

$$
\theta=\pi+\sum_{j=1}^{N}\left(a_{2 j-1} \cos [(2 j-1) \omega t / 3]+b_{2 j-1} \sin [(2 j-1) \omega t / 3]\right)
$$

while the asymmetric oscillations are given by (2.13) with $a_{0}$ near $\pi$ and $m=3$. The stability diagram for inverted, $3 T$-periodic oscillations is included in Figure 7, where the stability boundaries have the same significance as for the downward oscillations.

The stable regions for the normal and inverted oscillations of period $3 T$ overlap near $\omega=1.2$ and $\varepsilon=1.35$. The two types of oscillation appear to remain independent there, with the asymptotic state dependent on the initial conditions. The mean $\langle\theta\rangle$ for asymmetric oscillations lies either near zero for the downward oscillations or near $\pi$ for the inverted oscillations, with no stable solutions at intermediate values of $\langle\theta\rangle$.

\section{Running oscillations}

Running oscillatory solutions of (1.1) have the general form

$$
\theta=p \omega t / q+\sum_{k=0}^{N}\left[a_{k} \cos (k \omega t / q)+b_{k} \sin (k \omega t / q)\right]
$$

where $p$ and $q$ are integers. We consider here only $p=1$ and $q=1$, for which $\dot{\theta}$ is $T$-periodic (periodic refers to $\dot{\theta}$ throughout this section).

The stability diagram for running oscillations with mean angular velocity $\omega(p=q=1)$ is drawn in Figure 8, which extends over a much longer range of $\omega$ than the previous figures. The stability boundary $C_{1}$ is a turning-point locus for $T$-periodic oscillations. The oscillatory part of the motion decreases when $\varepsilon$ is increased along $C_{1}$, with $\dot{\theta}$ tending to $\omega$ and $a_{0}$ tending to $-\pi / 2$. The stability boundaries $C_{2}, C_{4}$, and $C_{8}$ are all period-doubling boundaries. Both $C_{4}$ and $C_{8}$ have been drawn, although they are almost indistinguishable in the figure. Comparison of Figure 8 with Figure 4 indicates that a stable running oscillation of period $T$ can be an alternative asymptotic state to a stable swinging oscillation of period $T$, provided that the initial angular velocity is sufficiently large. 


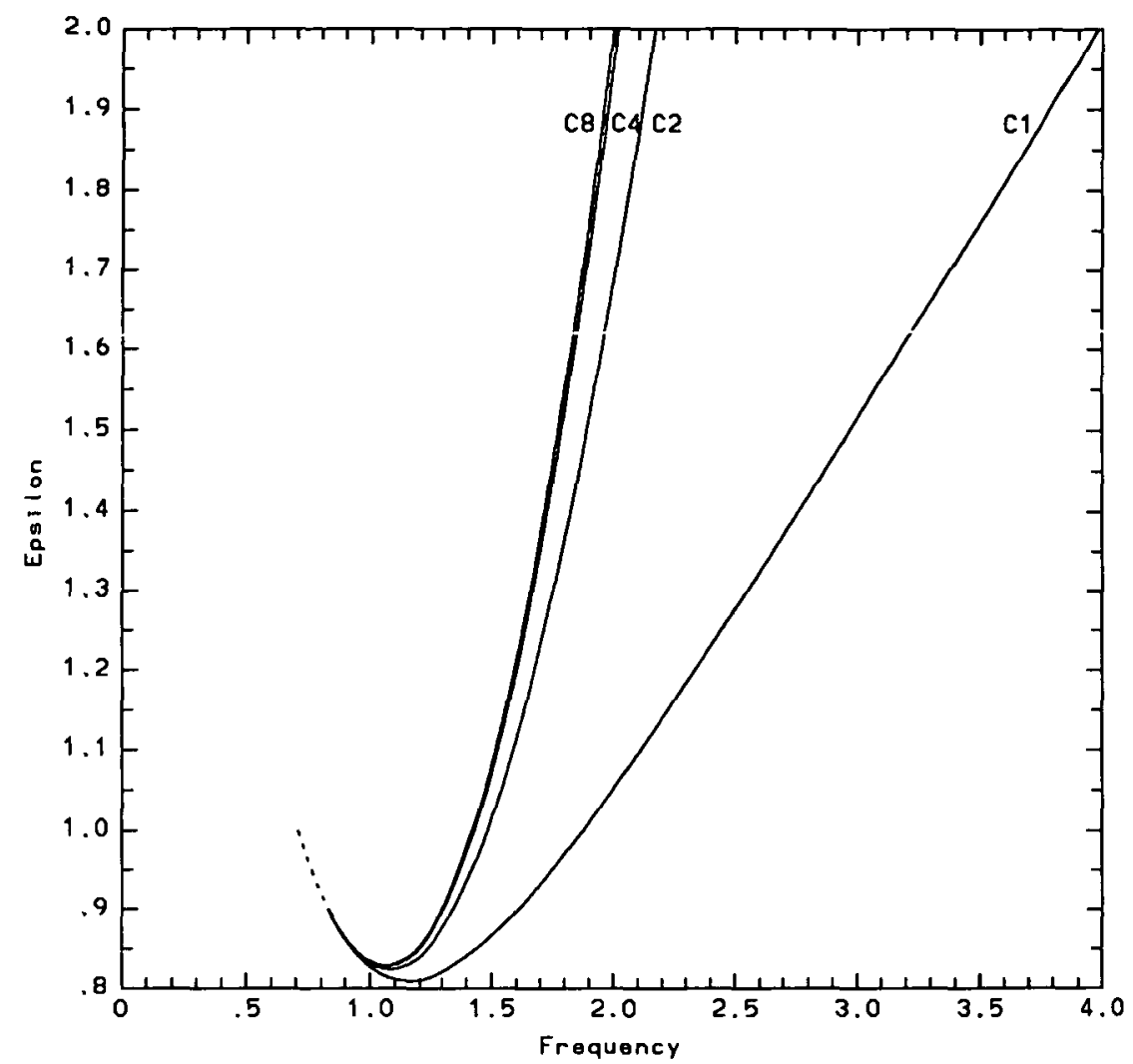

Figure 8. Stability boundaries for running oscillations of period $T, 2 T, 4 T, 8 T$, with mean angular velocity $\omega$ and $\delta=1 / 8$.

\section{Acknowledgement}

This work was supported in part by the Physical Oceanography, Applied Mathematics and Fluid Dynamics/Hydraulics programs of the National Science Foundation, NSF Grant OCE-81-17539, by the Office of Naval Research, Contract N00014-84-K-0137,4322318(430), and by the DARPA Univ. Res. Init. under Appl. and Comp. Math. Program Contract N0001486-K-0758 administered by the Office of Naval Research, and by a grant of computing time from the San Diego Supercomputer Center (sponsored by the National Science Foundation) [which was used for an accurate, systematic search of the asymptotic solutions in time to (1.1)]. 


\section{References}

[1] Peter J. Bryant and John W. Miles, “On a periodically-forced, weakly damped pendulum. Part 1: Applied torque", J. Austral. Math. Soc. Ser. B. 32 (1990) 1-22.

[2] Peter J. Bryant and John W. Miles, "On a periodically-forced, weakly damped pendulum. Part 3: Vertical forcing”, J. Austral. Math. Soc. Ser. B. 32 (1990) 42-60.

[3] Mitchell J. Feigenbaum, "Quantitative universality for a class of nonlinear transformations", J. Stat. Phys. 19 (1978) 25-52.

[4] D. D'Humieres, M. R. Beasley, B. A. Huberman, and A. Libchaber, "Chaotic states and routes to chaos in the forced pendulum", Phys. Rev. A 26 (1982) 3483-3496.

[5] John W. Miles, "Resonantly forced surface waves in a circular cylinder", J. Fluid Mech. 49 (1984) 15-31.

[6] John W. Miles, "Resonantly forced, nonlinear gravity waves in a shallow rectangular tank", Wave Motion 7 (1985) 291-297.

[7] John W. Miles, "Resonance and symmetry breaking for the pendulum", Physica D 31 (1988) 252-268.

[8] John W. Miles, "Directly forced oscillations of an inverted pendulum", Phys. Lett. A 133 (1988) 295-297.

[9] J. J. Stoker, Nonlinear Vibrations (Academic Press, New York, 1950) 103-109.

[10] J. W. Swift and K. Wiesenfeld, "Suppression of period doubling in symmetric systems", Phys. Rev. Lett. 52 (1984) 705-708. 